\title{
Constraining ESSnuSB neutrino flux by observing elastic scattering of neutrinos on electrons
}

\author{
Kaja Krhač ${ }^{1, *}$ \\ Center of Excellence for Advanced Materials and Sensing Devices, Ruđer Bošković Institute, \\ Bijenička cesta 54, 10000 Zagreb, Croatia \\ E-mail: kaja.krhac@irb.hr
}

ESSnuSB is a design study for a future experiment which will measure CP violation in the lepton sector at the $2^{\text {nd }}$ neutrino oscillation maximum. Since the experiment will observe muon neutrino to electron neutrino oscillations, it is important to measure interaction cross section of electron neutrinos with water. For this purpose, neutrino flux at near detector site must be precisely known. This work shows the progress done in constraining the ESSnuSB neutrino flux by considering the elastic scatterings of neutrinos on orbital electrons observed at the near water Cherenkov detector.

\footnotetext{
*** The European Physical Society Conference on High Energy Physics (EPS-HEP2021), ***

*** 26-30 July $2021 * * *$

*** Online conference, jointly organized by Universität Hamburg and the research center DESY ***

${ }^{1}$ On behalf of the ESSnuSB project.

${ }^{*}$ Speaker
} 


\section{Introduction}

The ESSnuSB project is a design study for an experiment to measure $\mathrm{CP}$ violation in the lepton sector at the $2^{\text {nd }}$ oscillation maximum. It aims to observe the difference in $v_{\mu} \rightarrow v_{e}$ oscillation probability.

Since muon (anti) neutrino beam will inevitably contain other neutrino flavours, a near water Cherenkov detector will be situated approximately 250 meters from the target to measure the neutrino flux, with the aim to reduce systematic uncertainties of the neutrino flux and measure neutrino-water interaction cross section [1][2].

\section{Neutrino flux}

Flux $L(t)$ can be determined from the cross section $\sigma$ and interaction rate $R(t)$ using 1 ,

$$
R(t)=\int_{0}^{\infty} \frac{d L(t)}{d E} \sigma(E) d E
$$

A study of a possibility to measure muon (anti)neutrino flux by observing the elastic scattering of neutrinos on electrons in the near water Cherenkov detector is being carried out. This process is well suited for neutrino flux measurement because its cross section can be very precisely calculated from theory ${ }^{1}$. However, the downside of the neutrino-electron scattering is that the cross section of this process is by 3-4 orders of magnitude smaller than that of the neutrino-nucleus scattering.

Expected fluxes were obtained using FLUKA [3] simulation of the ESSnuSB neutrino production facility. Prompt flux component of each neutrino flavour in neutrino beam is shown in Fig. 1a.

\section{Kinematics of elastic scattering}

It follows from kinematics of muon neutrino-electron elastic scattering that the energy of initial neutrino $E_{v}$ can be reconstructed by measuring the energy of outgoing electron $E_{e}$ and its escape angle $\theta_{e}$ (see Fig. 1b).

$$
E_{v}=\frac{m\left(E_{e}-m\right)}{m-E_{e}+\sqrt{E_{e}^{2}-m^{2}} \cos \theta_{e}}
$$

\section{Background rejection}

To measure the neutrino flux using scattering of neutrinos off electrons, one must discriminate these interactions from $v_{e} C C$ background. Following the analysis of MINERvA [4][5], parameter $\theta_{e}^{2} E_{e}$ (see Fig. 1b) was chosen as a discriminating variable. Its range is constrained by kinematics in the region $0 \leq \theta_{e}^{2} E_{e} \leq 2 m_{e}$. The results on a level of true Monte Carlo are shown in Fig. 2.

\footnotetext{
${ }^{1}$ Cross sections for processes in which neutrino scatters off a nucleus are difficult to calculate due to nuclear effects and little data in the energy region of interest.
} 


\section{Unfolding of the measured spectrum}

Measured spectrum $m$ depends on detector response $R$ and background $\beta$ according to Eq. 3 .

$$
m_{i}=\sum_{j}^{N} R_{i j} t_{j}+\beta_{i}, \quad \text { (3) } t_{i}=\sum_{j}^{N} R_{i j}^{-1}\left(m_{j}-\beta_{j}\right),
$$

The true spectrum $t$ is folded with the detector response [6] which is a characteristic of the detector. A response matrix obtained by taking the reconstructed energy of incoming neutrino as energy of outgoing electron is shown in Fig. 3. The true (initial) spectrum (Fig. 2) can be obtained by inverting relation 3, which is called unfolding. The results are shown in Fig. 4.

\section{Conclusion}

The ESSnuSB project is a design study which will measure CP violation in lepton sector at the $2^{\text {nd }}$ oscillation maximum by observing muon to electron (anti)neutrino oscillations. For this reason composition of the neutrino beam must be know precisely. A process well suited for measuring the neutrino flux is elastic neutrino-electron scattering. The energy of incoming neutrino can be very accurately determined if outgoing electron energy and an angle of outgoing electron with respect to incoming neutrino are known. Furthermore, this process can be successfully discriminated against the $v_{e} C C$ background using $\theta_{e}^{2} E_{e}$ variable. Finally, success of reconstruction depends on the detector response and background. Initial neutrino spectrum can be obtained using unfolding for any invertible response matrix. In this work only illustrative example of detector response was considered, but future plans are to include full detector response.

\section{Acknowledgements}

This project has received funding from the European Union's Horizon 2020 research and innovation programme under grant agreement No 777419 .

\section{References}

[1] B. Klicek, "The ESSnuSB project," PoS, vol. ICHEP2020, p. 152, 2021.

[2] M. Ghosh, "Overview of ESSnuSB experiment to measure $\delta_{C P}$," PoS, vol. NuFact2019, p. 038, 2020.

[3] A. Ferrari, P. R. Sala, A. Fasso, and J. Ranft, "FLUKA: A multi-particle transport code (Program version 2005)," 2005.

[4] J. Park et al., "Measurement of Neutrino Flux from Neutrino-Electron Elastic Scattering," Phys. Rev. D, vol. 93, no. 11, p. 112007, 2016.

[5] E. Valencia et al., "Constraint of the MINER $v$ A medium energy neutrino flux using neutrinoelectron elastic scattering," Phys. Rev. D, vol. 100, no. 9, p. 092001, 2019.

[6] G. Cowan, Statistical Data Analysis. Oxford science publications, Clarendon Press, 1998. 


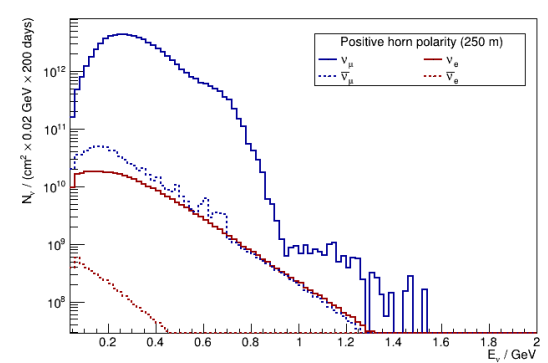

(a) Neutrino flux at $250 \mathrm{~m}$ from target.

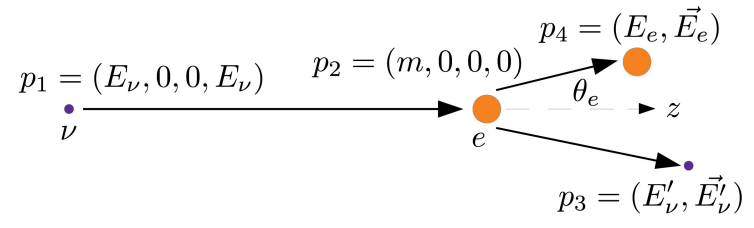

(b) Kinematics in laboratory system.

Figure 1: Neutrino flux and kinematics of neutrino-electron scattering

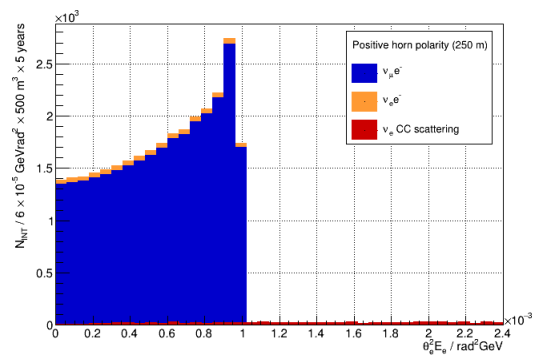

(a) Stack plot of number of expected events vs. $\theta_{e}^{2} E_{e}$ parameter.

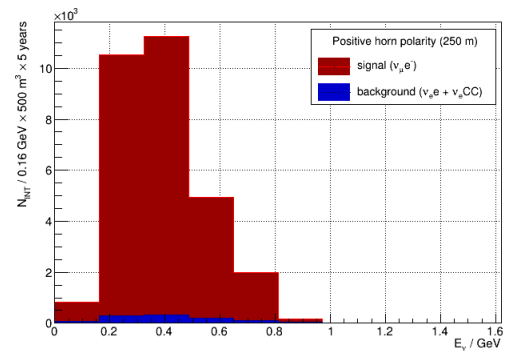

(b) Spectrum of events with an electron in the final state for which $\theta_{e}^{2} E_{e} \leq 2 m_{e}$.

Figure 2: Result of selection using true value of $\theta_{e}^{2} E_{e}$ variable for neutrino beam.

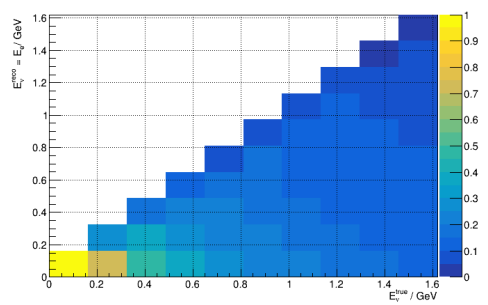

Figure 3: A response matrix when "measured" incoming neutrino energy is taken equal to $E_{e}$.

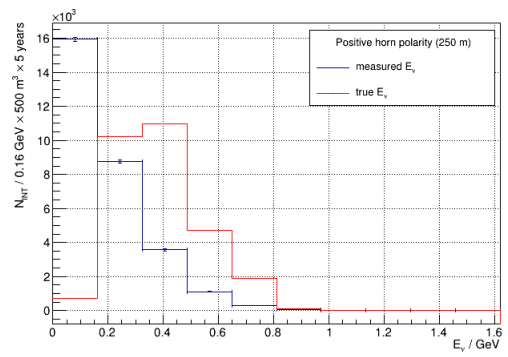

(a) Measured interaction spectrum compared to the true interaction spectrum.

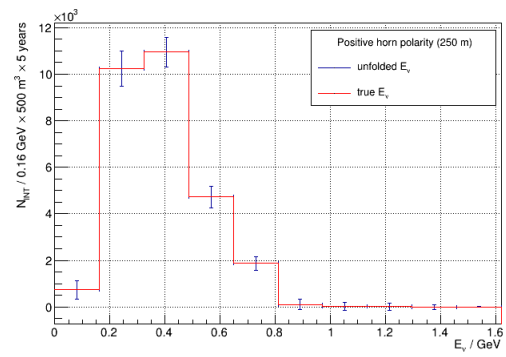

(b) Unfolded interaction spectrum compared to true interaction spectrum.

Figure 4: Results of unfolding neutrino spectrum produced with Fig. 2 and Fig. 3. Errors are statistical. 